\title{
Weak and strong approximation of semigroups on Hilbert spaces
}

\author{
R. Chill ${ }^{1}$ and A.F.M. ter Elst ${ }^{2}$
}

\begin{abstract}
For a sequence of uniformly bounded, degenerate semigroups on a Hilbert space, we compare various types of convergences to a limit semigroup. Among others, we show that convergence of the semigroups, or of the resolvents of the generators, in the weak operator topology, in the strong operator topology or in certain integral norms are equivalent under certain natural assumptions which are frequently met in applications.
\end{abstract}

September 2, 2016

AMS Subject Classification: 35B40, 47D03, 47A05, 65N30.

Keywords: Strong resolvent convergence, weak resolvent convergence, degenerate semigroup

\section{Home institutions:}

1. Institut für Analysis

TU Dresden

01062 Dresden

Germany
2. Department of Mathematics

University of Auckland

Private bag 92019

Auckland 1142

New Zealand 


\section{Introduction}

The subject of approximation of one-parameter semigroups of operators in various operator topologies is a fundamental topic in semigroup theory. The Trotter-Kato theorem for the approximation of a $C_{0}$-semigroup in the strong operator topology is a classical result which can be found in many textbooks. More recently, the question of approximation in the weak operator topology has been studied, too; see, for example, Król [Kró09], Eisner \& Sereny [ES10] and Furuya [Fur10]. The purpose of this article is to study the relation between convergence of a sequence of semigroups in the weak operator topology and the convergence in the strong operator topology. We concentrate on semigroups in Hilbert spaces whose generators are associated with $m$-sectorial forms. We show that convergence in the weak operator topology and in the strong operator topology are equivalent in the case where all involved semigroups are selfadjoint, while they are not equivalent in the general case, even when all semigroups are analytic and contractive on the same sector. In the case where all involved semigroups are analytic and contractive on the same sector we give additional conditions under which equivalence of convergence in the weak operator topology and the strong operator topology does hold. In fact, equivalence between the two types of convergences holds if in addition the semigroups generated by the real parts of the associated forms converge in the weak operator topology, or if a monotonicity condition holds which is for example satisfied in the context of the Galerkin approximation.

Motivated by applications to numerical analysis (the Galerkin approximation) or the stability of parabolic partial differential equations with respect to the underlying (unbounded) domain, we consider not only $C_{0}$-semigroups but general degenerate semigroups, that is, semigroups which are merely defined and strongly continuous on the open interval $(0, \infty)$, and bounded on the open interval $(0,1)$. In this more general context, it is for example possible to study the approximation of a $\left(C_{0^{-}}\right)$semigroup on an infinite dimensional Hilbert space by degenerate semigroups acting on finite dimensional subspaces. Due to the variational character of the applications which we describe in Sections 4, 5 and 6, convergence in the weak operator topology is often easy to establish while convergence in the strong operator topology is comparatively more involved, especially when compactness arguments (obtained by compact embeddings of domains of generators) are not at hand. In principle, the additional arguments which allow one to pass from convergence in the weak operator topology to convergence in the strong operator topology exist in a scattered way in the literature. In the case of the Galerkin method, these additional arguments are sometimes given, but sometimes the reader is left with a statement which does not give the full (strong) convergence properties, especially if one is only interested in abstract existence results for solutions of parabolic partial differential equations. The purpose of this article is to gather the arguments in an abstract context and to show that the equivalence between convergence in the weak and strong operator topology is a general principle independent from the concrete application in numerical analysis, the study of parabolic equations on varying domains or in homogenization. 


\section{Preliminaries on degenerate semigroups}

Let $X$ be a Banach space. We call a function $S:(0, \infty) \rightarrow \mathcal{L}(X), t \mapsto S_{t}$, a degenerate semigroup if

(i) $S$ is strongly continuous on $(0, \infty)$,

(ii) $S_{t+s}=S_{t} S_{s}$ for every $t, s \in(0, \infty)$, and

(iii) $\sup _{t \in(0,1)}\left\|S_{t}\right\|<\infty$.

It is an exercise to show, using properties (ii) and (iii) above, that every degenerate semigroup is exponentially bounded, that is, there exist constants $M \geq 0$ and $\omega \in \mathbb{R}$ such that

$$
\left\|S_{t}\right\| \leq M e^{\omega t}
$$

for all $t \in(0, \infty)$.

Let $A \subseteq X \times X$ be a graph in $X$. Then $-A$ is called the generator of a degenerate semigroup $S$ if there exists a $\omega \in \mathbb{R}$ such that $\lambda I+A$ is boundedly invertible for every $\lambda \in(\omega, \infty)$ and if

$$
(\lambda I+A)^{-1} x=\int_{0}^{\infty} e^{-\lambda t} S_{t} x d t
$$

for all $x \in X$. In particular, $\omega$ is chosen large enough so that the Laplace integral on the right-hand side converges. A crude estimate of the Laplace integral then yields that the pseudoresolvent $\lambda \mapsto(\lambda I+A)^{-1}$ satisfies the Hille-Yosida condition

$$
\left\|(\lambda-\omega)^{k}(\lambda I+A)^{-k}\right\| \leq M
$$

uniformly for all $k \in \mathbb{N}$ and $\lambda \in(\omega, \infty)$. There seems to be no characterisation of degenerate semigroups on Banach spaces known in the literature, for example solely in terms of the Hille-Yosida condition, the problem being that pseudoresolvents need not have dense range. However, there are two important situations in which one has a positive result.

The first is the situation of degenerate semigroups on reflexive spaces. If $A$ is a graph on a reflexive Banach space $X$, if there exists an $\omega \in \mathbb{R}$ such that $\lambda I+A$ is boundedly invertible for every $\lambda \in(\omega, \infty)$ and if the pseudoresolvent $\lambda \mapsto(\lambda I+A)^{-1}$ satisfies the Hille-Yosida condition above, then $-A$ is the generator of a degenerate semigroup $S$ for which, in addition, the limit

$$
P x:=\lim _{t \downarrow 0} S_{t} x
$$

exists for every $x \in X$ and defines a bounded projection $P$. Moreover, range $P=$ $\overline{\text { range }(\lambda I+A)^{-1}}$ and $\operatorname{ker} P=\operatorname{ker}(\lambda I+A)^{-1}$. In particular, the range and the kernel of $(\lambda I+A)^{-1}$ do not depend on $\lambda$, and $P$ is a projection onto the closure of the domain of $A$.

The second situation where a characterisation of the generator is available is the situation of analytic degenerate semigroups, that is, of degenerate semigroups which extend analytically to a sector of the form

$$
\Sigma_{\theta}:=\{z \in \mathbb{C} \backslash\{0\}:|\arg z|<\theta\}
$$


for some $\theta \in\left(0, \frac{\pi}{2}\right]$. If $A$ is a graph on a Banach space $X$, if there exist $\omega \in \mathbb{R}$ and $\theta \in\left(0, \frac{\pi}{2}\right]$ such that $\lambda I+A$ is boundedly invertible for every $\lambda \in \omega+\Sigma_{\frac{\pi}{2}+\theta}$, and if

$$
\sup _{\lambda \in \omega+\Sigma_{\frac{\pi}{2}+\theta}}\left\|(\lambda-\omega)(\lambda I+A)^{-1}\right\|<\infty
$$

then $-A$ generates a degenerate semigroup $S$ which extends analytically to a semigroup on the sector $\Sigma_{\theta}$. For the results stated above, see Arendt [Are01] or Baskakov [Bas04].

Analytic degenerate semigroups on Hilbert spaces are, for example, generated by graphs associated with closed, (quasi-) sectorial forms, and our main results concern indeed solely this particular situation, with the exception of Lemma [3.2, where we consider general analytic semigroups on Banach spaces. By a form on a Hilbert space $H$ we mean here a sesquilinear mapping $\mathfrak{a}: V \times V \rightarrow \mathbb{C}$, where the form domain $V$ is a linear subspace of $H$. We point out that the form domain $V$ need not be dense in $H$. The real part $\Re \mathfrak{a}$ of a form $\mathfrak{a}$ is defined by $(\Re \mathfrak{a})(u, v):=\frac{1}{2}(\mathfrak{a}(u, v)+\overline{\mathfrak{a}(v, u)})$, and similarly one may define the imaginary part which is, however, not used in this article. A form $\mathfrak{a}$ is called sectorial if there are $\theta \in\left(0, \frac{\pi}{2}\right)$ and $\gamma \in \mathbb{R}$ such that

$$
\mathfrak{a}(u)-\gamma\|u\|_{H}^{2} \in \overline{\Sigma_{\theta}}
$$

for all $u \in V$, where $\mathfrak{a}(u)=\mathfrak{a}(u, u)$. We call $\gamma$ a vertex of $\mathfrak{a}$. Finally, a sectorial form $\mathfrak{a}$ on $H$ is called closed if there exists an $\omega \in \mathbb{R}$ such that $(u, v) \mapsto(\Re \mathfrak{a})(u, v)+\omega(u, v)_{H}$ is a complete inner product on $V$.

For a closed, sectorial form we define the associated graph

$$
A:=\left\{(u, f) \in H \times H: u \in V \text { and } \mathfrak{a}(u, v)=(f, v)_{H} \text { for all } v \in V\right\} .
$$

Then this graph is $m$-sectorial in the sense that there is an $\omega>0$ such that $\lambda I+A$ is invertible and $\left\|\lambda(\lambda I+A)^{-1}\right\| \leq 1$ for every $\lambda \in \omega+\Sigma_{\theta+\frac{\pi}{2}}$. If $\mathfrak{a}$ is symmetric in the sense that $\mathfrak{a}=\Re \mathfrak{a}$, then the associated graph is self-adjoint. By applying [Kat80, Theorem VI.1.27] to the part of an $m$-sectorial graph in the closure of its domain one can see that every $m$-sectorial graph is associated to a closed, sectorial form.

\section{Semigroup convergence}

The first main result of this note is the following theorem for self-adjoint graphs and semigroups. It asserts that pointwise convergence of the resolvents in the weak operator topology and in the strong operator topology are equivalent, and that the same is true for pointwise convergence of semigroups in the weak operator topology and pointwise convergence of semigroups in the strong operator topology, uniformly for times in compact subsets of $(0, \infty)$.

Theorem 3.1. Let $H$ be a Hilbert space. For all $n \in \mathbb{N}$ let $A_{n}$ and $A$ be positive selfadjoint graphs on $H$. Let $S^{(n)}$ and $S$ be the degenerate semigroups generated by $-A_{n}$ and $-A$. Then the following are equivalent.

$$
\lim _{n \rightarrow \infty} S_{t}^{(n)}=S_{t} \text { in }(\mathcal{L}(H), \text { WOT }) \text { for all } t \in(0, \infty) .
$$


(ii) $\lim _{n \rightarrow \infty} \int_{0}^{T}\left(S_{t}^{(n)} f, g\right)_{H} d t=\int_{0}^{T}\left(S_{t} f, g\right)_{H} d t$ for all $T>0$ and $f, g \in H$.

(iii) $\lim _{n \rightarrow \infty} \int_{0}^{T}\left(S_{t}^{(n)} f, g(t)\right)_{H} d t=\int_{0}^{T}\left(S_{t} f, g(t)\right)_{H} d t$ for all $T>0, f \in H$ and $g \in$ $L_{1}([0, T], H)$.

(iv) $\lim _{n \rightarrow \infty} \int_{0}^{T}\left\|\left(S_{t}^{(n)}-S_{t}\right) f\right\|_{H} d t=0$ for all $T>0$ and $f \in H$.

(v) $\lim _{n \rightarrow \infty}\left(\lambda I+A_{n}\right)^{-1}=(\lambda I+A)^{-1}$ in $(\mathcal{L}(H)$, SOT $)$ for all $\lambda \in \mathbb{C}$ with $\operatorname{Re} \lambda>0$.

(vi) There exists a $\lambda \in \mathbb{C}$ with $\operatorname{Re} \lambda>0$ such that $\lim _{n \rightarrow \infty}\left(\lambda I+A_{n}\right)^{-1}=(\lambda I+A)^{-1}$ in $(\mathcal{L}(H), \mathrm{SOT})$.

(vii) $\lim _{n \rightarrow \infty}\left(\lambda I+A_{n}\right)^{-1}=(\lambda I+A)^{-1}$ in $(\mathcal{L}(H)$, WOT $)$ for all $\lambda \in \mathbb{C}$ with $\operatorname{Re} \lambda>0$.

(viii) There exists a set $D \subseteq \mathbb{C}$ with accumulation point in the open right half-plane $\{\mu \in$ $\mathbb{C}: \operatorname{Re} \mu>0\}$ such that $\lim _{n \rightarrow \infty}\left(\lambda I+A_{n}\right)^{-1}=(\lambda I+A)^{-1}$ in $(\mathcal{L}(H)$, WOT $)$ for all $\lambda \in D$.

(ix) There exists a $\lambda \in \mathbb{C} \backslash \mathbb{R}$ with $\operatorname{Re} \lambda>0$ such that $\lim _{n \rightarrow \infty}\left(\lambda I+A_{n}\right)^{-1}=(\lambda I+A)^{-1}$ in $(\mathcal{L}(H), \mathrm{WOT})$.

(x) For all $f \in H$ and $\delta, T>0$ with $\delta<T$ it follows that

$$
\lim _{n \rightarrow \infty} \sup _{t \in[\delta, T]}\left\|\left(S_{t}^{(n)}-S_{t}\right) f\right\|_{H}=0 .
$$

Theorem 3.1 is for self-adjoint graphs on Hilbert spaces. Statements (iv), (v), (vi) and $(\mathrm{x})$ are, however, also equivalent for general analytic degenerate semigroups on Banach spaces. This is the contents of the next lemma.

Lemma 3.2. Let $X$ be a Banach space. For all $n \in \mathbb{N}$ let $A_{n}$ and $A$ be graphs on $X$, such that $-A_{n}$ and $-A$ generate analytic degenerate semigroups $S^{(n)}$ and $S$, respectively. Assume there exists a $\theta \in\left(0, \frac{\pi}{2}\right]$ such that the degenerate semigroups $S^{(n)}$ and $S$ are uniformly bounded on the same sector $\Sigma_{\theta}$ with a bound independent of $n \in \mathbb{N}$. Then the following assertions are equivalent.

(i) $\quad \lim _{n \rightarrow \infty} \int_{0}^{T}\left\|\left(S_{t}^{(n)}-S_{t}\right) f\right\|_{X} d t=0$ for all $T>0$ and $f \in X$.

(ii) $\lim _{n \rightarrow \infty}\left(\lambda I+A_{n}\right)^{-1}=(\lambda I+A)^{-1}$ in $(\mathcal{L}(X)$, SOT $)$ for all $\lambda \in \mathbb{C}$ with $\operatorname{Re} \lambda>0$.

(iii) There exists a $\lambda \in \mathbb{C}$ with $\operatorname{Re} \lambda>0$ such that $\lim _{n \rightarrow \infty}\left(\lambda I+A_{n}\right)^{-1}=(\lambda I+A)^{-1}$ in $(\mathcal{L}(X), \mathrm{SOT})$.

(iv) For all $f \in X$ and $\delta, T>0$ with $\delta<T$ it follows that

$$
\lim _{n \rightarrow \infty} \sup _{t \in[\delta, T]}\left\|\left(S_{t}^{(n)}-S_{t}\right) f\right\|_{X}=0
$$

Proof. $($ i $) \Rightarrow\left(\right.$ ii) . This follows by taking Laplace transforms of the semigroups $S^{(n)}$ and $S$. (ii) $\Rightarrow$ (iii) . Trivial.

(iii) $\Rightarrow$ (ii) . First Condition (iii) implies that $\lim _{n \rightarrow \infty}\left(\lambda I+A_{n}\right)^{-k}=(\lambda I+A)^{-k}$ in $(\mathcal{L}(X)$, SOT $)$ for every $k \in \mathbb{N}$. Since the powers of $\left(\lambda I+A_{n}\right)^{-1}$ coincide, up to a scalar factor, with the derivatives of the holomorphic function $\mu \mapsto\left(\mu I+A_{n}\right)^{-1}$ at the point $\lambda$, Statement (ii) follows by an application of Vitali's theorem [AN00, Theorem 2.1]. (Compare also with [Are01, Remark 3.8] for a slightly different argument). 
(ii) $\Rightarrow$ (iv). This follows from Arendt Are01, Theorem 5.2]. A different proof is as follows. First it follows again from Vitali's theorem that $\lim _{n \rightarrow \infty}\left(\lambda I+A_{n}\right)^{-1}=(\lambda I+A)^{-1}$ in $(\mathcal{L}(X)$, SOT $)$ for all $\lambda \in \Sigma_{\frac{\pi}{2}+\theta}$. Secondly, for every $n \in \mathbb{N}, f \in X$ and $t>0$, one has the integral representation

$$
S_{t}^{(n)} f=\frac{1}{2 \pi i} \int_{\Gamma} e^{t \lambda}\left(\lambda I+A_{n}\right)^{-1} f d \lambda
$$

where $\Gamma$ is an appropriately chosen curve in $\Sigma_{\frac{\pi}{2}+\theta}$ connecting $e^{ \pm i \theta^{\prime}} \infty$ for some $\theta^{\prime} \in\left(\frac{\pi}{2}, \frac{\pi}{2}+\theta\right)$. Of course, this integral representation also holds when $S^{(n)}$ and $A_{n}$ are replaced by $S$ and $A$, respectively. The strong convergence of $\left(S_{t}^{(n)}\right)$, uniformly for $t$ in intervals of the form $[\delta, T]$ now follows from the locally uniform convergence of $\left(\lambda I+A_{n}\right)^{-1} f$ to $(\lambda I+A)^{-1} f$ and a rough estimate of the resolvents for large $\lambda$.

(iv) $\Rightarrow$ (i) . This follows from Lebesgue's dominated convergence theorem.

Now we turn to the proof of the main theorem of this section.

Proof of Theorem 3.1. As mentioned above, the equivalence of the statements (iv), (v), (vi) and (x) follows from Lemma 3.2.

(i) $\Rightarrow($ ii $)$. This follows from the Lebesgue dominated convergence theorem.

(ii) $\Rightarrow$ (iii) . Let $T>0$. It follows from Statement (ii) that

$$
\lim _{n \rightarrow \infty} \int_{0}^{T}\left(\left(S_{t}^{(n)}-S_{t}\right) f, \mathbb{1}_{[a, b]} g\right)_{H} d t=0
$$

for all $f, g \in H$ and $a, b \in \mathbb{R}$ with $0 \leq a<b \leq T$. Since the step functions are dense in $L_{1}([0, T], H)$ and the $S_{n}$ are contractive, Statement (iii) follows by a $3 \varepsilon$-argument.

(iii) $\Rightarrow$ (iv) . Let $T>0$ and $f \in H$. Then symmetry and the semigroup property give

$$
\begin{aligned}
\int_{0}^{T} \|\left(S_{t}^{(n)}-\right. & \left.S_{t}\right) f \|_{H}^{2} d t \\
& =\int_{0}^{T}\left(\left(S_{2 t}^{(n)}-S_{2 t}\right) f, f\right)_{H} d t-2 \operatorname{Re} \int_{0}^{T}\left(\left(S_{t}^{(n)}-S_{t}\right) f, S_{t} f\right)_{H} d t \\
& =\frac{1}{2} \int_{0}^{2 T}\left(\left(S_{t}^{(n)}-S_{t}\right) f, f\right)_{H} d t-2 \operatorname{Re} \int_{0}^{T}\left(\left(S_{t}^{(n)}-S_{t}\right) f, S_{t} f\right)_{H} d t
\end{aligned}
$$

for all $n \in \mathbb{N}$. Applying the hypothesis with $g(t)=f$ to the first term of (1) and with $g(t)=S_{t} f$ to the second term, one deduces that both terms on the right-hand side of (1) tend to 0 as $n \rightarrow \infty$. Hence $\lim _{n \rightarrow \infty} \int_{0}^{T}\left\|\left(S_{t}^{(n)}-S_{t}\right) f\right\|_{H}^{2} d t=0$. A simple application of the Cauchy-Schwarz inequality, using the contractivity of all involved semigroups, yields also convergence in the $L_{1}$-sense.

(iv) $\Leftrightarrow(\mathrm{v}) \Leftrightarrow(\mathrm{vi}) \Leftrightarrow(\mathrm{x})$. This is a special case of Lemma 3.2 . $(\mathrm{x}) \Rightarrow(\mathrm{i}),(\mathrm{v}) \Rightarrow(\mathrm{vii}) \Rightarrow(\mathrm{viii})$ and $($ vii) $\Rightarrow$ (ix) . Trivial.

(viii) $\Rightarrow$ (vii). This follows from Vitali's theorem.

(ix) $\Rightarrow($ vi $)$. Let $\lambda \in \mathbb{C} \backslash \mathbb{R}$ with $\operatorname{Re} \lambda>0$ be such that $\lim _{n \rightarrow \infty}\left(\lambda I+A_{n}\right)^{-1}=(\lambda I+A)^{-1}$ 
in $(\mathcal{L}(H)$, WOT). Let $f \in H$. Then

$$
\begin{aligned}
\lim _{n \rightarrow \infty}\left\|\left(\lambda I+A_{n}\right)^{-1} f\right\|_{H}^{2} & =\lim _{n \rightarrow \infty}\left(\left(\bar{\lambda} I+A_{n}\right)^{-1}\left(\lambda I+A_{n}\right)^{-1} f, f\right)_{H} \\
& =\lim _{n \rightarrow \infty}(\lambda-\bar{\lambda})^{-1}\left(\left(\left(\bar{\lambda} I+A_{n}\right)^{-1} f, f\right)_{H}-\left(\left(\lambda I+A_{n}\right)^{-1} f, f\right)_{H}\right) \\
& =(\lambda-\bar{\lambda})^{-1}\left(\left((\bar{\lambda} I+A)^{-1} f, f\right)_{H}-\left((\lambda I+A)^{-1} f, f\right)_{H}\right) \\
& =\left\|(\lambda I+A)^{-1} f\right\|_{H}^{2} .
\end{aligned}
$$

Then Statement (vi) is valid.

One might hope that the convergence in Statement $(\mathrm{x})$ of Theorem 3.1 is valid with $[\delta, T]$ replaced by $(0, T]$. However, a counterexample has been provided by Daners [Dan05. Example 6.7]. There is the following characterisation of uniform convergence on $(0, T]$ in the strong operator topology.

Lemma 3.3. Assume the assumptions and notation as in Lemma 3.2, and assume in addition that the Banach space $X$ is reflexive. Suppose the four equivalent statements in Lemma 3.2 are valid. For all $n \in \mathbb{N}$ let $P_{n}$ and $P$ be the projections given by

$$
P_{n} f:=\lim _{t \downarrow 0} S_{t}^{(n)} f \text { and } P f:=\lim _{t \downarrow 0} S_{t} f \quad(f \in X) .
$$

Then the following are equivalent.

(i) $\quad \lim _{n \rightarrow \infty} \sup _{t \in(0, T]}\left\|\left(S_{t}^{(n)}-S_{t}\right) f\right\|_{X}=0$ for all $T>0$ and $f \in X$.

(ii) There exists a $T>0$ such that $\lim _{n \rightarrow \infty} \sup _{t \in(0, T]}\left\|\left(S_{t}^{(n)}-S_{t}\right) f\right\|_{X}=0$ for all $f \in X$.

(iii) $\lim _{n \rightarrow \infty} P_{n}=P$ in $(\mathcal{L}(X), \mathrm{SOT})$.

Proof. (i) $\Rightarrow$ (ii) . Trivial.

(ii) $\Rightarrow$ (iii) . Since $\lim _{t \downarrow 0} S_{t}^{(n)} f=P_{n} f$ for all $f \in X$ and $n \in \mathbb{N}$, with a similar identity for $S$ and $P$, the implication (ii) $\Rightarrow$ (iii) follows by a $3 \varepsilon$-argument.

(iii) $\Rightarrow(\mathrm{i})$. It follows from the strong resolvent convergence and Are01, Theorem 4.2 (b)] that $\lim _{n \rightarrow \infty} \sup _{t \in(0, T]}\left\|\left(S_{t}^{(n)}-S_{t}\right) f\right\|_{X}=0$ for all $T>0$ and $f \in \overline{\operatorname{dom} A}$. Recall that $P$ is a projection onto $\overline{\operatorname{dom} A}$. Now let $T>0$ and $f \in X$. Then

$$
\begin{aligned}
\left\|S_{t}^{(n)} f-S_{t} f\right\|_{X} & =\left\|S_{t}^{(n)} P_{n} f-S_{t} P f\right\|_{X} \\
& \leq\left\|S_{t}^{(n)}\left(P_{n} f-P f\right)\right\|_{X}+\left\|\left(S_{t}^{(n)}-S_{t}\right) P f\right\|_{X} \\
& \leq M\left\|P_{n} f-P f\right\|_{X}+\left\|\left(S_{t}^{(n)}-S_{t}\right) P f\right\|_{X}
\end{aligned}
$$

for all $t \in(0, T]$ and $n \in \mathbb{N}$, where $M=\sup _{m \in \mathbb{N}, s \in(0, t]}\left\|S_{s}^{(m)}\right\|<\infty$, from which Statement (i) follows.

In Theorem 3.1[(ix) it is essential that $\lambda \notin \mathbb{R}$. In the next example there is convergence in $(\mathcal{L}(H)$, WOT $)$ for $\lambda=1$, but clearly not in $(\mathcal{L}(H)$, SOT). The example is part of [ES10, Example 2.3]. 
Example 3.4. Let $H=\ell_{2}$. For all $n \in \mathbb{N}$ define $U_{n} \in \mathcal{L}(H)$ by

$$
U_{n}\left(x_{1}, x_{2}, \ldots\right)=\left(x_{n+1}, \ldots, x_{2 n}, x_{1}, \ldots, x_{n}, x_{2 n+1}, \ldots\right)
$$

Then $U_{n}$ is self-adjoint and $\lim _{n \rightarrow \infty} U_{n}=0$ in $(\mathcal{L}(H)$, WOT $)$, but $\left(U_{n}\right)$ does not converge to 0 in $(\mathcal{L}(H)$, SOT $)$ since the $U_{n}$ are also unitary. Let $V_{n}=\left(1-\frac{1}{n}\right) U_{n}$ for all $n \in \mathbb{N}$. Then $\left\|V_{n}\right\|<1$ and the Cayley transform $A_{n}=\left(I+V_{n}\right)\left(I-V_{n}\right)^{-1} \in \mathcal{L}(H)$ is a positive self-adjoint operator. Moreover, $\left(I+A_{n}\right)^{-1}=\frac{1}{2}\left(I-V_{n}\right)=\frac{1}{2}\left(I-\left(1-\frac{1}{n}\right) U_{n}\right)$. So $\lim _{n \rightarrow \infty}\left(I+A_{n}\right)^{-1}=$ $\frac{1}{2} I=(I+A)^{-1}$ in $(\mathcal{L}(H)$, WOT $)$, where $A=I$ is a positive self-adjoint operator. However, $\lim _{n \rightarrow \infty}\left(I+A_{n}\right)^{-1}$ does not exist in $(\mathcal{L}(H), \mathrm{SOT})$, that is Statement (vi) in Theorem 3.1 is not valid.

We next present an example that symmetry of the generators in Theorem 3.1 cannot be replaced by $m$-sectoriality, even not with a uniform sector.

Example 3.5. Let $H$ be an infinite dimensional separable Hilbert space and let $S$ be the contraction semigroup defined by $S_{t}=e^{-t} I$ for all $t>0$. By [Kró09, Theorem 2.1] there exists a sequence $\left(S^{(n)}\right)_{n \in \mathbb{N}}$ of unitary $C_{0}$-groups on $H$ such that for all $T>0$ one has $\lim _{n \rightarrow \infty} S_{t}^{(n)}=S_{t}$ in $(\mathcal{L}(H)$, WOT $)$ uniformly for all $t \in(0, T]$. For all $n \in \mathbb{N}$ let $-B_{n}$ be the generator of $S^{(n)}$ and set $B=I$. Then, by taking Laplace transforms of the respective semigroups, $\lim _{n \rightarrow \infty}\left(\lambda I+B_{n}\right)^{-1}=(\lambda I+B)^{-1}$ in $(\mathcal{L}(H)$, WOT) for all $\lambda \in \mathbb{C}$ with $\operatorname{Re} \lambda>0$. Clearly, for each $t>0$ one does not have $\lim _{n \rightarrow \infty} S_{t}^{(n)}=S_{t}$ in $(\mathcal{L}(H)$, SOT), since the $S^{(n)}$ are isometric while $S$ is not. Hence for all $\lambda \in \mathbb{C}$ with $\operatorname{Re} \lambda>0$ one does not have $\lim _{n \rightarrow \infty}\left(\lambda I+B_{n}\right)^{-1}=(\lambda I+B)^{-1}$ in $(\mathcal{L}(H)$, SOT $)$, see Lemma 3.2.

The operator $B_{n}$ is not invertible in general, but $C_{n}=I+B_{n}$ is $m$-accretive and invertible for all $n \in \mathbb{N}$. Set $C=I+B=2 I$. Then $\lim _{n \rightarrow \infty}\left(\lambda I+C_{n}\right)^{-1}=(\lambda I+C)^{-1}$ in $(\mathcal{L}(H)$,WOT $)$ for all $\lambda \in \mathbb{C}$ with $\operatorname{Re} \lambda>0$. Moreover, for all $\lambda \in \mathbb{C}$ with $\operatorname{Re} \lambda>0$ one does not have $\lim _{n \rightarrow \infty}\left(\lambda I+C_{n}\right)^{-1}=(\lambda I+C)^{-1}$ in $(\mathcal{L}(H)$, SOT $)$. Finally, let $A_{n}=C_{n}^{1 / 2}$ for all $n \in \mathbb{N}$. Then $A_{n}$ is $m$-sectorial with vertex zero and semiangle $\frac{\pi}{4}$ by Kat80, Theorem V.3.35] and [ABHN01, Theorem 3.8.3]. Since

$$
\left(\lambda I+A_{n}\right)^{-1}=\frac{1}{\pi} \int_{0}^{\infty} \frac{\sqrt{\mu}}{\lambda^{2}+\mu}\left(\mu I+C_{n}\right)^{-1} d \mu
$$

for all $\lambda \in(0, \infty)$ by [Kat61, (A1)], it follows that $\lim _{n \rightarrow \infty}\left(\lambda I+A_{n}\right)^{-1}=(\lambda I+\sqrt{2} I)^{-1}$ in $(\mathcal{L}(H)$,WOT $)$ for all $\lambda \in(0, \infty)$. Note that $C_{n}=A_{n}^{2}$ and therefore $\left(I+C_{n}\right)^{-1}=$ $\left(i I+A_{n}\right)^{-1}\left(-i I+A_{n}\right)^{-1}$ for all $n \in \mathbb{N}$. So if $\lim _{n \rightarrow \infty}\left(i I+A_{n}\right)^{-1}$ converges in $(\mathcal{L}(H), \operatorname{SOT})$, then also $\lim _{n \rightarrow \infty}\left(-i I+A_{n}\right)^{-1}$ converges in $(\mathcal{L}(H)$, SOT) (compare with the argument in the proof of Lemma 3.2 using Vitali's theorem and the fact that $\pm i$ lie in the same component of analyticity of the resolvent) and hence $\lim _{n \rightarrow \infty}\left(I+C_{n}\right)^{-1}$ converges in $(\mathcal{L}(H)$, SOT), which is a contradiction. Thus Theorem 3.1 cannot be extended to $m$-sectorial operators.

Instead of considering the square roots $A_{n}=C_{n}^{\frac{1}{2}}$ one could also consider the fractional powers $A_{n}=C_{n}^{\alpha}$ for arbitrary $\alpha \in(0,1)$. The argument which allows one to pass from the convergence of the resolvents of $C_{n}$ in the weak operator topology to the convergence of the resolvents of $A_{n}$ in the weak operator topology (and back) then simply relies on a functional calculus representation of the resolvent of $A_{n}$ in terms of a contour integral over 
the resolvent of $C_{n}$ and vice versa [Haa06]. This means that the angle of sectoriality in the above counterexample can be chosen arbitrarily small.

A variant of Theorem 3.1 is true if, in addition, one also requires weak resolvent convergence for the real parts of the generators, or, more precisely, for the operators associated with the real parts of the involved forms. Under this additional assumption one again has that weak resolvent convergence implies strong resolvent convergence.

Theorem 3.6. Let $H$ be a Hilbert space. For all $n \in \mathbb{N}$ let $\mathfrak{a}_{n}$, $\mathfrak{a}$ be closed sectorial sesquilinear forms in $H$ with vertex zero. Let $A_{n}, A, R_{n}$ and $R$ be the m-sectorial graphs associated with $\mathfrak{a}_{n}, \mathfrak{a}, \Re \mathfrak{a}_{n}$ and $\Re \mathfrak{a}$, respectively. Suppose there exist $\lambda, \lambda^{\prime} \in \mathbb{C}$ with $\operatorname{Re} \lambda>0$, $\operatorname{Re} \lambda^{\prime}>0$ and $\lambda^{\prime} \notin \mathbb{R}$ such that

$$
\lim _{n \rightarrow \infty}\left(\lambda I+A_{n}\right)^{-1}=(\lambda I+A)^{-1} \quad \text { in }(\mathcal{L}(H), \text { WOT })
$$

and

$$
\lim _{n \rightarrow \infty}\left(\lambda^{\prime} I+R_{n}\right)^{-1}=\left(\lambda^{\prime} I+R\right)^{-1} \text { in }(\mathcal{L}(H), \text { WOT }) .
$$

Then $\lim _{n \rightarrow \infty}\left(I+A_{n}\right)^{-1}=(I+A)^{-1}$ in $(\mathcal{L}(H)$, SOT $)$ and, for all $f \in X$ and $\delta, T>0$ with $\delta<T$,

$$
\lim _{n \rightarrow \infty} \sup _{t \in[\delta, T]}\left\|\left(S_{t}^{(n)}-S_{t}\right) f\right\|_{X}=0
$$

Proof. It suffices to show that $\lim _{n \rightarrow \infty}\left(\lambda I+A_{n}\right)^{-1}=(\lambda I+A)^{-1}$ in $(\mathcal{L}(H)$, SOT $)$. Let $f \in H$. Write $u_{n}:=\left(\lambda I+A_{n}\right)^{-1} f$ and $u:=(\lambda I+A)^{-1} f$ for all $n \in \mathbb{N}$. Then $\lim _{n \rightarrow \infty} u_{n}=u$ weakly in $H$ by the assumed convergence of resolvents in the weak operator topology. Therefore we have to prove that $\lim _{n \rightarrow \infty} u_{n}=u$ (strongly) in $H$.

Let $n \in \mathbb{N}$. Then $\mathfrak{a}_{n}\left(u_{n}, v\right)+\lambda\left(u_{n}, v\right)_{H}=(f, v)_{H}$ for all $v \in V_{n}$. Choosing $v=u_{n}$ and taking the real part gives

$$
\operatorname{Re} \mathfrak{a}_{n}\left(u_{n}\right)+(\operatorname{Re} \lambda)\left\|u_{n}\right\|_{H}^{2}=\operatorname{Re}\left(f, u_{n}\right) .
$$

Similarly

$$
\operatorname{Re} \mathfrak{a}(u)+(\operatorname{Re} \lambda)\|u\|_{H}^{2}=\operatorname{Re}(f, u) .
$$

Since $\lim _{n \rightarrow \infty} u_{n}=u$ weakly in $H$, it follows from (2) that

$$
(\operatorname{Re} \lambda) \limsup _{n \rightarrow \infty}\left\|u_{n}\right\|_{H}^{2}=\limsup _{n \rightarrow \infty}\left(\operatorname{Re}\left(f, u_{n}\right)-\operatorname{Re} \mathfrak{a}_{n}\left(u_{n}\right)\right)=\operatorname{Re}(f, u)-\liminf _{n \rightarrow \infty} \operatorname{Re} \mathfrak{a}_{n}\left(u_{n}\right) .
$$

Now $\Re \mathfrak{a}_{n}$ and $\Re \mathfrak{a}$ are symmetric closed sesquilinear forms. Moreover, by assumption and by Theorem 3.1 (ix) $\Rightarrow(\mathrm{v})$ one has $\lim _{n \rightarrow \infty}\left(\lambda^{\prime} I+R_{n}\right)^{-1}=\left(\lambda^{\prime} I+R\right)^{-1}$ in $(\mathcal{L}(H)$, SOT). Using again that $\lim _{n \rightarrow \infty} u_{n}=u$ weakly in $H$, one deduces from Attouch [Att84, Theorem 3.26] (or for a shorter proof for forms, see Mosco [Mos94, Theorem 2.4.1]) the bound $(\Re \mathfrak{a})(u) \leq$ $\liminf _{n \rightarrow \infty}\left(\Re \mathfrak{a}_{n}\right)\left(u_{n}\right)$. Therefore

$$
(\operatorname{Re} \lambda) \limsup _{n \rightarrow \infty}\left\|u_{n}\right\|_{H}^{2}=\operatorname{Re}(f, u)-\liminf _{n \rightarrow \infty} \operatorname{Re} \mathfrak{a}_{n}\left(u_{n}\right) \leq \operatorname{Re}(f, u)-\operatorname{Re} \mathfrak{a}(u)=(\operatorname{Re} \lambda)\|u\|_{H}^{2},
$$

where we used (3) in the last step. So $\lim _{n \rightarrow \infty} u_{n}=u$ in $H$ and the convergence of resolvents in the strong operator topology follows. The remaining assertion on the convergence of semigroups follows from Lemma 3.2 . 
We finish this section by presenting a theorem in which we deal with a single form $\mathfrak{a}$ which does not have to be symmetric and where the approximation is connected to a space approximation of the form domain. It is not a corollary to the main theorem (Theorem 3.1) nor is it an immediate consequence of Theorem 3.6. We rather give a variant of the proof of the latter which does not use the Mosco convergence hidden in the references to Attouch [Att84] or Mosco [Mos94].

Theorem 3.7. Let $V$ and $H$ be Hilbert spaces such that $V$ is continuously embedded in $H$. Let $\mathfrak{a}: V \times V \rightarrow \mathbb{C}$ be a closed, sectorial, sesquilinear form. Let $A$ be the m-sectorial graph in $H$ associated with $\mathfrak{a}$. Let $\left(V_{n}\right)$ be an increasing sequence of closed subspaces of $V$ such that $\bigcup_{n \in \mathbb{N}} V_{n}$ is dense in $V$. For all $n \in \mathbb{N}$ let $\mathfrak{a}_{n}=\left.\mathfrak{a}\right|_{V_{n} \times V_{n}}$. Further let $A_{n}$ be the $m$-sectorial graph in $H$ associated with $\mathfrak{a}_{n}$. Let $S^{(n)}$ and $S$ be the semigroups generated by $-A_{n}$ and $-A$, respectively. Then

$$
\lim _{n \rightarrow \infty} \sup _{t \in(0, T]}\left\|\left(S_{t}^{(n)}-S_{t}\right) f\right\|_{H}=0
$$

for every $T>0$ and every $f \in H$.

Proof. Without loss of generality we may assume that the form $\mathfrak{a}$ is coercive, that is, that there exists a $\mu>0$ such that $\mu\|u\|_{V}^{2} \leq \operatorname{Re} \mathfrak{a}(u)$ for all $u \in V$.

Let $f \in H$. Let $n \in \mathbb{N}$. Set $u_{n}=\left(I+A_{n}\right)^{-1} f$. Then $u_{n} \in V_{n}$ and

$$
\mathfrak{a}\left(u_{n}, v\right)+\left(u_{n}, v\right)_{H}=(f, v)_{H}
$$

for all $v \in V_{n}$. Choose $v=u_{n}$. Then $\operatorname{Re} \mathfrak{a}\left(u_{n}\right)+\left\|u_{n}\right\|_{H}^{2}=\operatorname{Re}\left(f, u_{n}\right)_{H} \leq\|f\|_{H}\left\|u_{n}\right\|_{H}$. So $\left\|u_{n}\right\|_{H} \leq\|f\|_{H}$ and $\mu\left\|u_{n}\right\|_{V}^{2} \leq \operatorname{Re} \mathfrak{a}\left(u_{n}\right) \leq\|f\|_{H}^{2}$. Therefore the sequence $\left(u_{n}\right)_{n \in \mathbb{N}}$ is bounded in $V$. Passing to a subsequence if necessary, there exists a $u \in V$ such that $\lim _{n \rightarrow \infty} u_{n}=u$ weakly in $V$. Let $m \in \mathbb{N}$ and $v \in V_{m}$. Then

$$
\mathfrak{a}\left(u_{n}, v\right)+\left(u_{n}, v\right)_{H}=(f, v)_{H}
$$

for all $n \in \mathbb{N}$ with $n \geq m$. Take the limit $n \rightarrow \infty$. Then

$$
\mathfrak{a}(u, v)+(u, v)_{H}=(f, v)_{H} .
$$

Since $\bigcup_{n \in \mathbb{N}} V_{n}$ is dense in $V$ one deduces that (44) is valid for all $v \in V$. So $u \in D(A)$ and $u=(I+A)^{-1} f$. Since

$$
\begin{aligned}
\limsup _{n \rightarrow \infty}\left\|u_{n}\right\|_{H}^{2} & =\limsup _{n \rightarrow \infty}\left(\operatorname{Re}\left(f, u_{n}\right)_{H}-\operatorname{Re} \mathfrak{a}\left(u_{n}\right)\right) \\
& =\operatorname{Re}(f, u)_{H}-\liminf _{n \rightarrow \infty} \operatorname{Re} \mathfrak{a}\left(u_{n}\right) \\
& \leq \operatorname{Re}(f, u)_{H}-\operatorname{Re} \mathfrak{a}(u)=\|u\|_{H}^{2}
\end{aligned}
$$

it follows that $\lim _{n \rightarrow \infty} u_{n}=u$ in $H$. So $\lim _{n \rightarrow \infty}\left(I+A_{n}\right)^{-1}=(I+A)^{-1}$ in $(\mathcal{L}(H)$, SOT $)$. In particular, the four equivalent statements of Lemma 3.2 hold.

For every $n \in \mathbb{N}$ the limits

$$
P_{n} f:=\lim _{t \downarrow 0} S_{t}^{(n)} f \text { and } P f:=\lim _{t \downarrow 0} S_{t} f
$$


exist and define projections onto the closures (in $H$ ) of the domains of the graphs $A_{n}$ and $A$, respectively, and thus by [Kat80, Theorem VI.2.1 ii)] onto the closures of $V_{n}$ and $V$ in $H$. Since the graphs $A_{n}$ and $A$ are associated with forms, one can easily see from their definition that the projections $P_{n}$ and $P$ are orthogonal. Using again that $\left(V_{n}\right)$ is increasing and that $\bigcup_{n \in \mathbb{N}} V_{n}$ is dense in $V$, we find that $\lim _{n \rightarrow \infty} P_{n}=P$ in $(\mathcal{L}(H), \mathrm{SOT})$. The claim follows from Lemma 3.2 and Lemma 3.3 .

The situation of Theorem 3.7 has a flavour of the situation of a monotonically decreasing sequence of forms, if one can speak of monotonicity in the context of sectorial forms. If the conclusion was strong resolvent convergence of the operators $A_{n}$, it may be seen as a generalisation of Simon [Sim78, Theorems 3.2 and 4.1]. By Lemma 3.2, the strong resolvent convergence is equivalent to the convergence of the semigroups in the strong operator topology, uniformly in times from compact subsets of $(0, \infty)$. The uniform convergence up to $t=0$ is an additional feature of Theorem 3.7. The situation of Theorem 3.7 is somewhat opposite to the situation of a monotonically increasing sequence of forms for which strong resolvent convergence of associated operators follows from Kato Kat80, Theorem VIII.3.13a] and Simon [Sim78, Theorems 3.1 and 4.1] in the symmetric case and from Batty \& ter Elst [BtE14] in a somewhat more general case of sectorial forms.

\section{Galerkin approximation}

One popular situation in which an analytic $C_{0^{-}}$or degenerate semigroup is approximated by degenerate semigroups arises in the numerical analysis of parabolic partial differential equations, namely in the Galerkin approximation, that is, the space discretization via finite element spaces. It is not necessary to state a separate corollary for this situation since Theorem 3.7 is precisely designed for it. Given two Hilbert spaces $V$ and $H$ such that $V$ is continuously embedded in $H$, and given a closed, sectorial, sesquilinear form $\mathfrak{a}: V \times V \rightarrow \mathbb{C}$, it suffices to chose an increasing sequence $\left(V_{n}\right)$ of finite dimensional subspaces of $V$ (finite

element spaces) such that $\bigcup_{n \in \mathbb{N}} V_{n}$ is dense in $V$. Then Theorem 3.7 asserts that the semigroups generated by the graphs $A_{n}$ associated with the forms $\mathfrak{a}_{n}:=\left.\mathfrak{a}\right|_{V_{n} \times V_{n}}$ converge in the strong operator topology to the semigroup generated by the graph $A$ associated with $\mathfrak{a}$, uniformly for times in intervals of the form $(0, T]$.

In some textbooks on linear and nonlinear analysis, the Galerkin approximation is used as a method of proof of existence of solutions of abstract elliptic, parabolic or hyperbolic equations. The approximate solutions living in finite dimensional subspaces of the energy space usually fulfill some a priori estimates, that is, they live in bounded subsets of the form domain or in a function space with values in the form domain. Then an argument using weak compactness allows one to find limit points (in a weak topology), and these limit points are shown to be solutions of the original equation. The question of convergence of the approximate solutions in a norm topology is, however, not systematically discussed. In Drábek-Milota [DM13, Proposition 7.2.41] (see also Evans [Eva90, Theorem 2 in Chapter 2], the strong convergence of approximate solutions of a (nonlinear) stationary problem is explicitly stated, while this is not done in the case of approximate solutions of a (nonlinear) gradient system in reflexive spaces [DM13, Theorem 8.2.5]. A statement on strong 
convergence is also missing in Evans [Eva98, Theorem 3 in $\S 7.1 .2]$ in the context of an abstract linear parabolic problem, even under restrictive assumptions on the finite elements. Yet, the strong convergence of approximate solutions in the Galerkin approximation of abstract parabolic equations is known and Theorem 3.7 is not new in this context: see the monograph by Dautray-Lions [DL92, Remark 5 in Section XVIII.3, p. 520] with uniform convergence in time on bounded intervals of $(0, \infty)$ or the survey by Fujita-Suzuki [FS91, Theorem 7.1] with uniform convergence in time on compact intervals of $(0, \infty)$, in order to mention only two references.

Note, however, that the subspaces $V_{n}$ in Theorem 3.7 do not have to be finite dimensional.

\section{$5 \quad$ Elliptic and parabolic problems on varying domains}

In this section we illustrate Theorems 3.6 and 3.7 by considering a sequence of diffusion equations on varying open sets $\Omega_{n}$ which converge monotonically from below to an open set $\Omega$. We provide new proofs for the next results, which have been studied also in Simon Sim78, Example 1 and Theorem 4.1 in Section 4]. In the following, given an open set $\Omega \subseteq \mathbb{R}^{d}$, we consider the Sobolev space $H_{0}^{1}(\Omega)$ as a subspace of $H^{1}\left(\mathbb{R}^{d}\right)$ by identifying functions in $H_{0}^{1}(\Omega)$ with functions in $H^{1}\left(\mathbb{R}^{d}\right)$ which are equal to 0 almost everywhere on $\mathbb{R}^{d} \backslash \Omega$, and similarly we consider the space $L_{2}(\Omega)$ as a subspace of $L_{2}\left(\mathbb{R}^{d}\right)$.

Theorem 5.1. Let $\left(\Omega_{n}\right)_{n \in \mathbb{N}}$ be an increasing sequence of open subsets of $\mathbb{R}^{d}$ and let $\Omega:=$ $\bigcup_{n \in \mathbb{N}} \Omega_{n}$. Let $a \in L_{\infty}\left(\Omega ; \mathbb{C}^{d \times d}\right)$ be uniformly elliptic in the sense that there exists $\eta>0$ such that

$$
\sum_{i, j=1}^{d} a_{i j}(x) \xi_{j} \overline{\xi_{i}} \geq \eta|\xi|^{2} \text { for every } \xi \in \mathbb{C}^{d} \text { and } x \in \Omega .
$$

For all $n \in \mathbb{N}$ let $u_{0, n} \in L_{2}\left(\Omega_{n}\right) \subseteq L_{2}(\Omega)$ and $u_{0} \in L_{2}(\Omega)$. Further, for all $n \in \mathbb{N}$ let $u_{n} \in C\left([0, \infty) ; L_{2}\left(\Omega_{n}\right)\right)$ and $u \in C\left([0, \infty) ; L_{2}(\Omega)\right)$ be the solutions of the diffusion equations

$$
\begin{aligned}
\partial_{t} u_{n}-\operatorname{div}\left(a(x) \nabla u_{n}\right) & =0 & & \text { in }(0, \infty) \times \Omega_{n}, \\
u_{n} & =0 & & \text { on }(0, \infty) \times \partial \Omega_{n}, \\
u_{n}(0, \cdot) & =u_{0, n} & & \text { in } \Omega_{n},
\end{aligned}
$$

and

$$
\begin{aligned}
\partial_{t} u-\operatorname{div}(a(x) \nabla u) & =0 & & \text { in }(0, \infty) \times \Omega, \\
u & =0 & & \text { on }(0, \infty) \times \partial \Omega, \\
u(0, \cdot) & =u_{0} & & \text { in } \Omega,
\end{aligned}
$$

respectively. If $\lim _{n \rightarrow \infty}\left\|u_{0, n}-u_{0}\right\|_{L_{2}\left(\mathbb{R}^{d}\right)}=0$, then

$$
\lim _{n \rightarrow \infty} \sup _{t \in[0, T]}\left\|u_{n}(t)-u(t)\right\|_{L_{2}\left(\mathbb{R}^{d}\right)}=0 .
$$

Proof. For all $n \in \mathbb{N}$ we consider the sectorial sesquilinear form $\mathfrak{a}_{n}: H_{0}^{1}\left(\Omega_{n}\right) \times H_{0}^{1}\left(\Omega_{n}\right) \rightarrow \mathbb{C}$ defined by $\mathfrak{a}_{n}(u, v)=\int_{\Omega_{n}}(a(x) \nabla u) \cdot \overline{\nabla v}$ and we denote by $A_{n}$ the sectorial graph on $L_{2}(\Omega)$ associated to $\mathfrak{a}_{n}$. Similarly, we define the form $\mathfrak{a}$ on $H_{0}^{1}(\Omega)$ and the associated operator $A$, 
by replacing $\Omega_{n}$ by $\Omega$ in the above definition. Observe that $\mathfrak{a}_{n}$ is the restriction of the form $\mathfrak{a}$ to the space $H_{0}^{1}\left(\Omega_{n}\right)$. Observe in addition that $u_{n}(t)=S_{t}^{(n)} u_{0, n}$ for all $t>0$, where $S^{(n)}$ is the semigroup generated by $-A_{n}$, and similarly $u(t)=S_{t} u_{0}$ for all $t>0$, where $S$ is the semigroup generated by $-A$.

Since the sequence $\left(\Omega_{n}\right)$ is monotonically increasing to $\Omega$, it is easy to see that $C_{c}^{\infty}(\Omega)$ is a subspace of $\bigcup_{n} H_{0}^{1}\left(\Omega_{n}\right)$ and thus the latter space is dense in $H_{0}^{1}(\Omega)$. The claim then follows from Theorem 3.7 .

As a consequence of Theorem 5.1 and Lemma 3.2, we obtain strong convergence of solutions of elliptic problems, that is, convergence of resolvents in the strong operator topology.

Corollary 5.2. Let $\left(\Omega_{n}\right)_{n \in \mathbb{N}}$ be an increasing sequence of open subsets of $\mathbb{R}^{d}$ and let $\Omega:=$ $\bigcup_{n \in \mathbb{N}} \Omega_{n}$. Fix $\lambda>0$ and $f \in L_{2}(\Omega)$. For all $n \in \mathbb{N}$ let $u_{n} \in H_{0}^{1}\left(\Omega_{n}\right)$ be the weak solution of the Dirichlet problem

$$
\begin{aligned}
\lambda u_{n}-\Delta u_{n}=f & \text { in } \Omega_{n}, \\
u_{n}=0 & \text { on } \partial \Omega_{n} .
\end{aligned}
$$

Further, let $u \in H_{0}^{1}(\Omega)$ be the weak solution of

$$
\begin{aligned}
\lambda u-\Delta u=f & \text { in } \Omega, \\
u=0 & \text { on } \partial \Omega .
\end{aligned}
$$

Then $\lim _{n \rightarrow \infty}\left\|u_{n}-u\right\|_{L_{2}\left(\mathbb{R}^{d}\right)}=0$.

There is a characterisation for convergence of resolvents of the Dirichlet Laplacian or resolvents of more general operators as in Theorem 5.1 in the strong operator topology. One defines that a sequence $\left(H_{0}^{1}\left(\Omega_{n}\right)\right)_{n \in \mathbb{N}}$ of Sobolev spaces converges in the sense of Mosco [Mos69] if the following two conditions are valid:

(i) if $u_{n} \rightarrow u$ weakly in $H^{1}\left(\mathbb{R}^{d}\right)$ and $u_{n} \in H_{0}^{1}\left(\Omega_{n}\right)$ for all $n \in \mathbb{N}$, then $u \in H_{0}^{1}(\Omega)$, and

(ii) for every $u \in H_{0}^{1}(\Omega)$ there exists a sequence $\left(u_{n}\right)$ with $u_{n} \in H_{0}^{1}\left(\Omega_{n}\right)$ for all $n \in \mathbb{N}$ and $u_{n} \rightarrow u$ in $H^{1}\left(\mathbb{R}^{d}\right)$;

see, for example, Dan05, Assumption 6.2]. Then convergence of resolvents in the strong operator topology is valid if and only if the sequence $\left(H_{0}^{1}\left(\Omega_{n}\right)\right)$ converges to $H_{0}^{1}(\Omega)$ in the sense of Mosco, see Daners [Dan03, Theorem 5.3] for the implication ' $\Leftarrow$ ' and for the implication ' $\Rightarrow$ ' see Attouch [Att84, Theorem 3.26] or Mosco [Mos94, Theorem 2.4.1].

Let us sketch a proof of the implication that Mosco convergence of the Sobolev spaces implies strong resolvent convergence. In fact, it is not difficult to prove the convergence $\left(\lambda I+A_{n}\right)^{-1} \rightarrow(\lambda I+A)^{-1}$ in the weak operator topology whenever $\lambda>0$. Similarly, $\left(\lambda I+R_{n}\right)^{-1} \rightarrow(\lambda I+R)^{-1}$ in the weak operator topology, where $R_{n}$ and $R$ are the operators associated with the real parts $\Re \mathfrak{a}_{n}$ and $\Re \mathfrak{a}$, respectively, simply because the latter sesquilinear forms have a similar structure as the forms $\mathfrak{a}_{n}$ and $\mathfrak{a}$ and the same arguments apply. Once, the two convergences in the weak operator topology are shown, the convergence in the strong operator topology follows from Theorem 3.6 . 
Of course, by Lemma 3.2 again, the convergence of resolvents in the strong operator topology implies convergence of the semigroups in the strong operator topology, uniformly in time intervals of the form $[\delta, T]$ with $\delta, T>0$. This is weaker than the convergence of the semigroups in the strong operator topology, uniformly in time intervals of the form $(0, T]$, as stated in Theorem [5.1. In the situation of merely Mosco convergence of the spaces $H_{0}^{1}\left(\Omega_{n}\right)$ to $H_{0}^{1}(\Omega)$, one cannot expect uniform convergence on $(0, T]$ in general, as the example from [Dan05, Example 6.7] shows.

The problem of stability of solutions of elliptic equations with respect to the domain has been studied extensively in the works by Bucur [Buc99], Bucur \& Butazzo [BB02], Bucur \& Varchon [BV00], Daners [Dan03], Daners, Hauer \& Dancer [DHD15], Arrieta \& Barbatis [AB14, Arendt \& Daners [AD07, [AD08], Biegert \& Daners [BD06], Dal Maso \& Toader [DMT96], Sa Ngiamsunthorn [SN12a], [SN12b] and Mugnolo, Nittka \& Post [MNP13].

\section{Homogenization on (unbounded) open sets}

We next illustrate Theorem 3.1 and consider the classical problem of homogenization of second-order elliptic operators with periodic coefficients.

For all $k, l \in\{1, \ldots, d\}$ let $c_{k l}: \mathbb{R}^{d} \rightarrow \mathbb{R}$ be measurable and bounded. Suppose that these coefficients are

(i) symmetric, that is, $c_{k l}=c_{l k}$ for all $k, l \in\{1, \ldots, d\}$,

(ii) periodic, that is, $c_{k l}(x+\gamma)=c_{k l}(x)$ for all $k, l \in\{1, \ldots, d\}, x \in \mathbb{R}^{d}$ and $\gamma \in \mathbb{Z}^{d}$, and

(iii) uniformly elliptic, that is, there exists a $\mu>0$ such that $\sum_{k, l=1}^{d} c_{k l}(x) \xi_{k} \overline{\xi_{l}} \geq \mu|\xi|^{2}$ for all $x \in \mathbb{R}^{d}$ and $\xi \in \mathbb{C}^{d}$.

For all $\varepsilon>0$ and $k, l \in\{1, \ldots, d\}$ define $c_{k l}^{(\varepsilon)}: \mathbb{R}^{d} \rightarrow \mathbb{R}$ by $c_{k l}^{(\varepsilon)}(x)=c_{k l}\left(\frac{1}{\varepsilon} x\right)$. Let $\Omega \subseteq \mathbb{R}^{d}$ be open. We emphasise that we do not assume that $\Omega$ is bounded. Let $V$ be a closed subspace of $H^{1}(\Omega)$ which contains $C_{c}^{\infty}(\Omega)$. For all $\varepsilon>0$ let $A_{\varepsilon}$ be the self-adjoint operator in $L_{2}(\Omega)$ associated to the form $\mathfrak{a}_{\varepsilon}: V \times V \rightarrow \mathbb{C}$ defined by

$$
\mathfrak{a}_{\varepsilon}(u, v)=\int_{\Omega} \sum_{k, l=1}^{d} c_{k l}^{(\varepsilon)}\left(\partial_{k} u\right) \overline{\partial_{l} v}
$$

We shall prove that there exists a positive self-adjoint operator $\widehat{A}$ in $L_{2}(\Omega)$ such that

$$
\lim _{\varepsilon \downarrow 0}\left(\lambda I+A_{\varepsilon}\right)^{-1}=(\lambda I+\widehat{A})^{-1}
$$

in the strong operator topology for all $\lambda \in \mathbb{C}$ with $\operatorname{Re} \lambda>0$. In fact, we determine the operator $\widehat{A}$ and we only need to prove convergence in the weak operator topology.

An explicit description of $\widehat{A}$ is as follows. Consider the space $H_{\mathrm{per}}^{1}\left(\mathbb{R}^{d}\right)$ of all functions $u \in H_{\text {loc }}^{1}\left(\mathbb{R}^{d}\right)$ which satisfy $u(x+\gamma)=u(x)$ for a.e. $x \in \mathbb{R}^{d}$ and all $\gamma \in \mathbb{Z}^{d}$. For all $j \in\{1, \ldots, d\}$ there exists a $\chi_{j} \in H_{\text {per }}^{1}\left(\mathbb{R}^{d}\right)$ such that

$$
\int_{[0,1]^{d}} \sum_{k, l=1}^{d} c_{k l}\left(\partial_{k} \chi_{j}\right) \overline{\partial_{l} v}=-\int_{[0,1]^{d}} \sum_{k, l=1}^{d} c_{j l} \overline{\partial_{l} v}
$$


for all $v \in H_{\text {per }}^{1}\left(\mathbb{R}^{d}\right)$. Then $\chi_{j} \in L_{\infty}\left(\mathbb{R}^{d}\right)$ by Stampacchia [Sta60, Teorema 4.1] since $c_{j l} \in L_{p}$ with $p>d$. For all $k, l \in\{1, \ldots, d\}$ define

$$
\hat{c}_{k l}=\int_{[0,1]^{d}} c_{k l}-\sum_{j=1}^{d} \int_{[0,1]^{d}} c_{k j} \partial_{j} \chi_{l} .
$$

It follows from Bensoussan, Lions \& Papanicolau [BLP78, Remark 1.2.6] that there exists a $\mu^{\prime}>0$ such that $\sum_{k, l=1}^{d} \hat{c}_{k l} \xi_{k} \bar{\xi}_{l} \geq \mu^{\prime}|\xi|^{2}$ for all $\xi \in \mathbb{C}^{d}$. Let $\widehat{A}$ be the operator in $L_{2}(\Omega)$ associated to the form $\mathfrak{a}: V \times V \rightarrow \mathbb{C}$ defined by

$$
\mathfrak{a}(u, v)=\int_{\Omega} \sum_{k, l=1}^{d} \hat{c}_{k l}\left(\partial_{k} u\right) \overline{\partial_{l} v} .
$$

The alluded theorem is the following.

Theorem 6.1. Let $\lambda \in \mathbb{C}$ with $\operatorname{Re} \lambda>0$. Then

$$
\lim _{\varepsilon \downarrow 0}\left(\lambda I+A_{\varepsilon}\right)^{-1}=(\lambda I+\widehat{A})^{-1} \text { in }(\mathcal{L}(H), \mathrm{SOT}) .
$$

Proof. Let $f \in L_{2}(\Omega)$ and let $\lambda \in \mathbb{C}$ with $\operatorname{Re} \lambda>0$. Let $\left(\varepsilon_{n}\right)$ be a sequence of positive real numbers such that $\lim _{n \rightarrow \infty} \varepsilon_{n}=0$. Let $n \in \mathbb{N}$. Set $u_{n}=\left(\lambda I+A_{\varepsilon_{n}}\right)^{-1} f$. Then

$$
\int_{\Omega} \sum_{k, l=1}^{d} c_{k l}^{\left(\varepsilon_{n}\right)}\left(\partial_{k} u_{n}\right) \overline{\partial_{l} v}+\lambda \int_{\Omega} u_{n} \bar{v}=\int_{\Omega} f \bar{v}
$$

for all $v \in V$. Choosing $v=u_{n}$ gives

$$
\mu \int_{\Omega}\left|\nabla u_{n}\right|^{2}+(\operatorname{Re} \lambda) \int_{\Omega}\left|u_{n}\right|^{2} \leq \operatorname{Re} \int_{\Omega} f \overline{u_{n}} \leq\|f\|_{L_{2}(\Omega)}\left\|u_{n}\right\|_{L_{2}(\Omega)} .
$$

So $\left\|u_{n}\right\|_{L_{2}(\Omega)} \leq(\operatorname{Re} \lambda)^{-1}\|f\|_{L_{2}(\Omega)}$ and $\mu \int_{\Omega}\left|\nabla u_{n}\right|^{2} \leq(\operatorname{Re} \lambda)^{-1}\|f\|_{L_{2}(\Omega)}^{2}$. Hence the sequence $\left(u_{n}\right)_{n \in \mathbb{N}}$ is bounded in $V$. For all $k \in\{1, \ldots, d\}$ and $n \in \mathbb{N}$ define

$$
w_{k n}=\sum_{l=1}^{d} c_{k l}^{\left(\varepsilon_{n}\right)} \partial_{l} u_{n}
$$

Then the sequence $\left(w_{k n}\right)_{n \in \mathbb{N}}$ is bounded in $L_{2}(\Omega)$ for all $k \in\{1, \ldots, d\}$. Passing to a subsequence, if necessary, there exist $u \in V$ and $w_{1}, \ldots, w_{d} \in L_{2}(\Omega)$ such that $\lim _{n \rightarrow \infty} u_{n}=$ $u$ weakly in $V$ and $\lim _{n \rightarrow \infty} w_{k n}=w_{k}$ weakly in $L_{2}(\Omega)$ for all $k \in\{1, \ldots, d\}$.

By (6) one has

$$
\sum_{k=1}^{d} \int_{\Omega} w_{k n} \overline{\partial_{k} v}+\lambda \int_{\Omega} u_{n} \bar{v}=\int_{\Omega} f \bar{v}
$$

for all $v \in V$ and $n \in \mathbb{N}$. Take the limit $n \rightarrow \infty$. Then

$$
\sum_{k=1}^{d} \int_{\Omega} w_{k} \overline{\partial_{k} v}+\lambda \int_{\Omega} u \bar{v}=\int_{\Omega} f \bar{v}
$$

for all $v \in V$. We next determine the $w_{i}$, which requires some work. 
Let $i \in\{1, \ldots, d\}$. Let $\varphi \in C_{c}^{\infty}(\Omega)$. For all $n \in \mathbb{N}$ define $\chi_{i}^{(n)} \in H_{\text {loc }}^{1}\left(\mathbb{R}^{d}\right)$ by $\chi_{i}^{(n)}(x)=$ $\chi_{i}\left(\frac{1}{\varepsilon_{n}} x\right)$. We denote by $\pi_{k}: \mathbb{R}^{d} \rightarrow \mathbb{R}$ the $k$-th coordinate function for all $k \in\{1, \ldots, d\}$. Let $n \in \mathbb{N}$. Then $\varphi\left(\pi_{i}-\varepsilon_{n} \chi_{i}^{(n)}\right) \in H_{0}^{1}(\Omega) \subseteq V$. Moreover, (6) gives

$$
\begin{aligned}
& \int_{\Omega} f \overline{\varphi\left(\pi_{i}-\varepsilon_{n} \chi_{i}^{(n)}\right)}= \int_{\Omega} \sum_{l=1}^{d} w_{l n} \overline{\partial_{l}\left(\varphi\left(\pi_{i}-\varepsilon_{n} \chi_{i}^{(n)}\right)\right)}+\lambda \int_{\Omega} u_{n} \bar{\varphi}\left(\pi_{i}-\varepsilon_{n} \chi_{i}^{(n)}\right) \\
&=\int_{\Omega} \sum_{l=1}^{d} w_{l n} \overline{\partial_{l} \varphi}\left(\pi_{i}-\varepsilon_{n} \chi_{i}^{(n)}\right)+\int_{\Omega} \sum_{l=1}^{d} w_{l n} \bar{\varphi} \partial_{l}\left(\pi_{i}-\varepsilon_{n} \chi_{i}^{(n)}\right) \\
&+\lambda \int_{\Omega} u_{n} \bar{\varphi}\left(\pi_{i}-\varepsilon_{n} \chi_{i}^{(n)}\right) .
\end{aligned}
$$

The second term on the right hand side of (8) can be rewritten as

$$
\begin{aligned}
& \int_{\Omega} \sum_{l=1}^{d} w_{l n} \bar{\varphi} \partial_{l}\left(\pi_{i}-\varepsilon_{n} \chi_{i}^{(n)}\right) \\
& \quad=\sum_{k, l=1}^{d} \int_{\Omega} c_{k l}^{\left(\varepsilon_{n}\right)}\left(\partial_{k} u_{n}\right) \bar{\varphi} \partial_{l}\left(\pi_{i}-\varepsilon_{n} \chi_{i}^{(n)}\right) \\
& \quad=\sum_{k, l=1}^{d} \int_{\Omega} c_{k l}^{\left(\varepsilon_{n}\right)}\left(\partial_{k}\left(\bar{\varphi} u_{n}\right)\right) \partial_{l}\left(\pi_{i}-\varepsilon_{n} \chi_{i}^{(n)}\right)-\sum_{k, l=1}^{d} \int_{\Omega} c_{k l}^{\left(\varepsilon_{n}\right)} u_{n} \overline{\partial_{k} \varphi} \partial_{l}\left(\pi_{i}-\varepsilon_{n} \chi_{i}^{(n)}\right) .
\end{aligned}
$$

Note that $\bar{\varphi} u_{n} \in H_{0}^{1}(\Omega) \subseteq H^{1}\left(\mathbb{R}^{d}\right)$ by extending the function with zero. Define $v_{n} \in$ $H^{1}\left(\mathbb{R}^{d}\right)$ by $v_{n}(x)=\left(\bar{\varphi} u_{n}\right)\left(\varepsilon_{n} x\right)$. Then $v_{n}$ has compact support. The first term can be simplified since

$$
\sum_{k, l=1}^{d} \int_{\Omega} c_{k l}^{\left(\varepsilon_{n}\right)}\left(\partial_{k}\left(\bar{\varphi} u_{n}\right)\right) \partial_{l}\left(\pi_{i}-\varepsilon_{n} \chi_{i}^{(n)}\right)=\frac{1}{\varepsilon_{n}} \sum_{k, l=1}^{d} \int_{\mathbb{R}^{d}} c_{k l}\left(\partial_{k} v_{n}\right) \partial_{l}\left(\pi_{i}-\chi_{i}\right)=0
$$

by (5). So (8) gives

$$
\begin{aligned}
\int_{\Omega} f \overline{\varphi\left(\pi_{i}-\varepsilon_{n} \chi_{i}^{(n)}\right)}=\int_{\Omega} \sum_{l=1}^{d} w_{l n} \overline{\partial_{l} \varphi}\left(\pi_{i}-\varepsilon_{n} \chi_{i}^{(n)}\right)-\sum_{k, l=1}^{d} \int_{\Omega} c_{k l}^{\left(\varepsilon_{n}\right)} u_{n} \overline{\partial_{k} \varphi} \partial_{l}\left(\pi_{i}-\varepsilon_{n} \chi_{i}^{(n)}\right) \\
+\lambda \int_{\Omega} u_{n} \bar{\varphi}\left(\pi_{i}-\varepsilon_{n} \chi_{i}^{(n)}\right) .
\end{aligned}
$$

Now take the limit $n \rightarrow \infty$. Since $\chi_{i} \in L_{\infty}\left(\mathbb{R}^{d}\right)$ it follows that

$$
\lim _{n \rightarrow \infty} \int_{\Omega} f \overline{\varphi\left(\pi_{i}-\varepsilon_{n} \chi_{i}^{(n)}\right)}=\int_{\Omega} f \bar{\varphi} \pi_{i} .
$$

Also

$$
\left|\int_{\Omega} w_{l n}\left(\partial_{l} \varphi\right) \chi_{i}^{(n)}\right| \leq\left\|\chi_{i}\right\|_{\infty}\left\|w_{l n}\right\|_{L_{2}(\Omega)}\left\|\partial_{l} \varphi\right\|_{L_{2}(\Omega)}
$$

for all $n \in \mathbb{N}$ and the sequence $\left(w_{l n}\right)_{n \in \mathbb{N}}$ is bounded in $L_{2}(\Omega)$. So

$$
\lim _{n \rightarrow \infty} \int_{\Omega} \sum_{l=1}^{d} w_{l n} \overline{\partial_{l} \varphi}\left(\pi_{i}-\varepsilon_{n} \chi_{i}^{(n)}\right)=\int_{\Omega} \sum_{l=1}^{d} w_{l} \overline{\partial_{l} \varphi} \pi_{i} .
$$


In order to evaluate the limit of the second term on the right hand side of (9), we need a lemma.

Lemma 6.2. Let $\tau: \mathbb{R}^{d} \rightarrow \mathbb{R}$ be measurable and periodic, i.e. $\tau(x+\gamma)=\tau(x)$ for all $x \in \mathbb{R}^{d}$ and $\gamma \in \mathbb{Z}^{d}$. Suppose that $\left.\tau\right|_{[0,1]^{d}} \in L_{2}\left([0,1]^{d}\right)$. Let $\Omega \subseteq \mathbb{R}^{d}$ be open. Let $v, v_{1}, v_{2}, \ldots \in L_{2}(\Omega)$ and $K \subseteq \Omega$ compact. Suppose that $\lim _{n \rightarrow \infty} v_{n}=v$ in $L_{2}(\Omega)$ and $\operatorname{supp} v_{n} \subseteq K$ for all $n \in \mathbb{N}$. Let $\left(\varepsilon_{n}\right)$ be a sequence of positive real numbers such that $\lim _{n \rightarrow \infty} \varepsilon_{n}=0$. Then

$$
\lim _{n \rightarrow \infty} \int_{\Omega} \tau\left(\frac{1}{\varepsilon_{n}} x\right) v_{n}=\left(\int_{[0,1]^{d}} \tau\right) \int_{\Omega} v
$$

Proof. The proof is similar to the proof in the one dimensional case in Bra02, Example 2.4].

We continue with the proof of Theorem 6.1. Let $k \in\{1, \ldots, d\}$. Then $\lim u_{n} \overline{\partial_{k} \varphi}=$ $u \overline{\partial_{k} \varphi}$ weakly in $V$, hence weakly in $H^{1}\left(\mathbb{R}^{d}\right)$. Since $\operatorname{supp} \overline{\partial_{k} \varphi}$ is a compact subset of $\Omega$, it follows that $\lim u_{n} \overline{\partial_{k} \varphi}=u \overline{\partial_{k} \varphi}$ in $L_{2}(\Omega)$. Apply Lemma 6.2 with $\tau=c_{k l} \partial_{l}\left(\pi_{i}-\chi_{i}\right)$. Then

$$
\begin{aligned}
\lim _{n \rightarrow \infty} \sum_{k, l=1}^{d} \int_{\Omega} c_{k l}^{\left(\varepsilon_{n}\right)} u_{n} \overline{\partial_{k} \varphi} \partial_{l}\left(\pi_{i}-\varepsilon_{n} \chi_{i}^{(n)}\right) & =\sum_{k, l=1}^{d}\left(\int_{[0,1]^{d}} c_{k l} \partial_{l}\left(\pi_{i}-\chi_{i}\right)\right) \int_{\Omega} u \overline{\partial_{k} \varphi} \\
& =-\sum_{k=1}^{d} \hat{c}_{k i} \int_{\Omega}\left(\partial_{k} u\right) \bar{\varphi}
\end{aligned}
$$

where we used the definition of the homogenized coefficients and integrated by parts.

The last term in (9) is easy and

$$
\lim _{n \rightarrow \infty} \lambda \int_{\Omega} u_{n} \bar{\varphi}\left(\pi_{i}-\varepsilon_{n} \chi_{i}^{(n)}\right)=\lambda \int_{\Omega} u \bar{\varphi} \pi_{i}
$$

Combining the limits, it follows from (9) that

$$
\int_{\Omega} f \bar{\varphi} \pi_{i}=\int_{\Omega} \sum_{l=1}^{d} w_{l} \overline{\partial_{l} \varphi} \pi_{i}+\sum_{k=1}^{d} \hat{c}_{k i} \int_{\Omega}\left(\partial_{k} u\right) \bar{\varphi}+\lambda \int_{\Omega} u \bar{\varphi} \pi_{i}
$$

Next, choosing $v=\varphi \pi_{i}$ in (7) gives

$$
\int_{\Omega} f \bar{\varphi} \pi_{i}=\sum_{k=1}^{d} \int_{\Omega} w_{k} \overline{\partial_{k}\left(\varphi \pi_{i}\right)}+\lambda \int_{\Omega} u \bar{\varphi} \pi_{i}=\sum_{k=1}^{d} \int_{\Omega} w_{k} \overline{\partial_{k} \varphi} \pi_{i}+\int_{\Omega} w_{i} \bar{\varphi}+\lambda \int_{\Omega} u \bar{\varphi} \pi_{i} .
$$

Hence

$$
\int_{\Omega} w_{i} \bar{\varphi}=\sum_{k=1}^{d} \hat{c}_{k i} \int_{\Omega}\left(\partial_{k} u\right) \bar{\varphi}
$$

This equality is valid for all $\varphi \in C_{c}^{\infty}(\Omega)$. So $w_{i}=\sum_{k=1}^{d} \hat{c}_{k i} \partial_{k} u$. Then (7) gives

$$
\sum_{k, l=1}^{d} \int_{\Omega} \hat{c}_{k l}\left(\partial_{k} u\right) \overline{\partial_{l} v}+\lambda \int_{\Omega} u \bar{v}=\int_{\Omega} f \bar{v}
$$

for all $v \in V$. Therefore $u \in \operatorname{dom} \widehat{A}$ and $(\lambda I+\widehat{A}) u=f$. 
We showed that $\lim _{n \rightarrow \infty}\left(\lambda I+A_{\varepsilon_{n}}\right)^{-1}=(\lambda I+\widehat{A})^{-1}$ in the weak operator topology for all $\lambda \in \mathbb{C}$ with $\operatorname{Re} \lambda>0$. Then Theorem 3.1 gives that the limit is also valid in the strong operator topology.

We emphasise once again that we do not assume that $\Omega$ is bounded. Strong resolvent convergence with bounded $\Omega$ has been obtained in Bensoussan, Lions \& Papanicolau [BLP78, Theorem 1.5.1] using much more work involving additional correctors. Strong resolvent convergence on $\mathbb{R}^{d}$ has been proved in [ZP05, Theorem 1.7] and for bounded $\Omega$ with Dirichlet or Neumann boundary conditions in [ZP05, Theorems 2.3 and 2.8]. A strong convergence of a slightly different nature can be found in Allaire All92.

In the case $V=H_{0}^{1}(\Omega)$ one has the following consequence of Theorems 6.1 and 3.1 .

Corollary 6.3. For all $\varepsilon \in(0,1]$ let $u_{0, \varepsilon} \in L_{2}(\Omega)$ and let $u_{0} \in L_{2}(\Omega)$. Further, for all $\varepsilon \in(0,1]$ let $u_{\varepsilon} \in C\left(\left([0, \infty) ; L_{2}(\Omega)\right)\right.$ and $u \in C\left(\left([0, \infty) ; L_{2}(\Omega)\right)\right.$ be the solutions of the diffusion equations

$$
\begin{aligned}
\partial_{t} u_{n}+A_{\varepsilon} u_{\varepsilon} & =0 & & \text { in }(0, \infty) \times \Omega, \\
u_{\varepsilon} & =0 & & \text { on }(0, \infty) \times \partial \Omega, \\
u_{n}(0, \cdot) & =u_{0, \varepsilon} & & \text { in } \Omega,
\end{aligned}
$$

and

$$
\begin{array}{rlrl}
\partial_{t} u+\widehat{A} u & =0 & & \text { in }(0, \infty) \times \Omega, \\
u=0 & & \text { on }(0, \infty) \times \partial \Omega, \\
u(0, \cdot)=u_{0} & \text { in } \Omega,
\end{array}
$$

respectively. Let $\delta, T>0$ with $\delta \leq T$. If $\lim _{\varepsilon \downarrow 0}\left\|u_{0, \varepsilon}-u_{0}\right\|_{L_{2}(\Omega)}=0$, then

$$
\lim _{\varepsilon \downarrow 0} \sup _{t \in[\delta, T]}\left\|u_{n}(t)-u(t)\right\|_{L_{2}(\Omega)}=0 .
$$

A similar corollary is valid for Neumann boundary conditions.

\section{Acknowledgements}

The second-named author is most grateful for the hospitality extended to him during a fruitful stay at the TU Dresden. He wishes to thank the Institut für Analysis for financial support. Part of this work is supported by an NZ-EU IRSES counterpart fund and the Marsden Fund Council from Government funding, administered by the Royal Society of New Zealand. Part of this work is supported by the EU Marie Curie IRSES program, project 'AOS', No. 318910.

\section{References}

[All92] G. Allaire. Homogenization and two-scale convergence. SIAM J. Math. Anal., 23(6):1482-1518, 1992.

[Are01] W. Arendt. Approximation of degenerate semigroups. Taiwanese J. Math., 5:279-295, 2001. 
[ABHN01] W. Arendt, C. J. K. Batty, M. Hieber, and F. Neubrander. Vector-valued Laplace Transforms and Cauchy Problems, volume 96 of Monographs in Mathematics. Birkhäuser, Basel, 2001.

[AD07] W. Arendt and D. Daners. Uniform convergence for elliptic problems on varying domains. Math. Nachr., 280(1-2):28-49, 2007.

[AD08] W. Arendt and D. Daners. Varying domains: stability of the Dirichlet and the Poisson problem. Discrete Contin. Dyn. Syst., 21(1):21-39, 2008.

[AN00] W. Arendt and N. Nikolski. Vector-valued holomorphic functions revisited. Math. Z., 234(4):777-805, 2000.

[AB14] J. M. Arrieta and G. Barbatis. Stability estimates in $H_{0}^{1}$ for solutions of elliptic equations in varying domains. Math. Methods Appl. Sci., 37(2):180-186, 2014.

[Att84] H. Attouch. Variational convergence for functions and operators. Applicable Mathematics Series. Pitman (Advanced Publishing Program), Boston, MA, 1984.

[Bas04] A. G. Baskakov. Theory of representations of Banach algebras, and abelian groups and semigroups in the spectral analysis of linear operators. Sovrem. Mat. Fundam. Napravl., 9:3-151 (electronic), 2004.

[BtE14] C. J. K. Batty and A. F. M. ter Elst. On series of sectorial forms. J. Evol. Equ., 14(1):29-47, 2014.

[BLP78] A. Bensoussan, J.-L. Lions, and G. Papanicolaou. Asymptotic analysis for periodic structures, volume 5 of Studies in Mathematics and its Applications. North-Holland Publishing Co., Amsterdam-New York, 1978.

[BD06] M. Biegert and D. Daners. Local and global uniform convergence for elliptic problems on varying domains. J. Differential Equations, 223(1):1-32, 2006.

[Bra02] A. Braides. Г-convergence for beginners, volume 22 of Oxford Lecture Series in Mathematics and its Applications. Oxford University Press, Oxford, 2002.

[Buc99] D. Bucur. Characterization for the Kuratowski limits of a sequence of Sobolev spaces. J. Differential Equations, 151(1):1-19, 1999.

[BB02] D. Bucur and G. Buttazzo. Variational methods in some shape optimization problems. Appunti dei Corsi Tenuti da Docenti della Scuola. [Notes of Courses Given by Teachers at the School]. Scuola Normale Superiore, Pisa, 2002.

[BV00] D. Bucur and N. Varchon. Boundary variation for a Neumann problem. Ann. Scuola Norm. Sup. Pisa Cl. Sci. (4), 29(4):807-821, 2000.

[DMT96] G. Dal Maso and R. Toader. A capacity method for the study of Dirichlet problems for elliptic systems in varying domains. Rend. Sem. Mat. Univ. Padova, 96:257-277, 1996. 
[Dan03] D. Daners. Dirichlet problems on varying domains. J. Differential Equations, 188(2):591-624, 2003.

[Dan05] D. Daners. Perturbation of semi-linear evolution equations under weak assumptions at initial time. J. Differential Equations, 210(2):352-382, 2005.

[DHD15] D. Daners, D. Hauer, and E. N. Dancer. Uniform convergence of solutions to elliptic equations on domains with shrinking holes. Adv. Differential Equations, 20(5-6):463-494, 2015.

[DL92] R. Dautray and J.-L. Lions. Mathematical analysis and numerical methods for science and technology. Vol. 5. Springer-Verlag, Berlin, 1992. Evolution problems. I, With the collaboration of Michel Artola, Michel Cessenat and Hélène Lanchon, Translated from the French by Alan Craig.

[DM13] P. Drábek and J. Milota. Methods of nonlinear analysis. Birkhäuser Advanced Texts: Basler Lehrbücher. [Birkhäuser Advanced Texts: Basel Textbooks]. Birkhäuser/Springer Basel AG, Basel, second edition, 2013. Applications to differential equations.

[ES10] T. Eisner and A. Serény. On the weak analogue of the Trotter-Kato theorem. Taiwanese J. Math., 14(4):1411-1416, 2010.

[Eva90] L. C. Evans. Weak convergence methods for nonlinear partial differential equations, volume 74 of CBMS Regional Conference Series in Mathematics. Published for the Conference Board of the Mathematical Sciences, Washington, DC; by the American Mathematical Society, Providence, RI, 1990.

[Eva98] L. C. Evans. Partial Differential Equations, volume 19 of Graduate Studies in Mathematics. American Mathematical Society, Providence, RI, 1998.

[FS91] H. Fujita and T. Suzuki. Evolution problems. In Handbook of numerical analysis, Vol. II, Handb. Numer. Anal., II, pages 789-928. North-Holland, Amsterdam, 1991.

[Fur10] K. Furuya. Trotter-Kato theorem for weak convergence on Hilbert space cases. Adv. Math. Sci. Appl., 20(1):143-152, 2010.

[Haa06] M. Haase. The functional calculus for sectorial operators, volume 169 of Operator Theory: Advances and Applications. Birkhäuser Verlag, Basel, 2006.

[Kat61] T. Kato. Fractional powers of dissipative operators. J. Math. Soc. Japan, 13:246-274, 1961.

[Kat80] T. Kato. Perturbation theory for linear operators, volume 132 of Grundlehren der mathematischen Wissenschaften. Springer-Verlag, Berlin, 1980.

[Kró09] S. Król. A note on approximation of semigroups of contractions on Hilbert spaces. Semigroup Forum, 79(2):369-376, 2009. 
[Mos69] U. Mosco. Convergence of convex sets and of solutions of variational inequalities. Advances in Math., 3:510-585, 1969.

[Mos94] U. Mosco. Composite media and asymptotic Dirichlet forms. J. Funct. Anal., 123(2):368-421, 1994.

[MNP13] D. Mugnolo, R. Nittka, and O. Post. Norm convergence of sectorial operators on varying Hilbert spaces. Oper. Matrices, 7(4):955-995, 2013.

[SN12a] P. Sa Ngiamsunthorn. Domain perturbation for parabolic equations. Bull. Aust. Math. Soc., 85(1):174-176, 2012.

[SN12b] P. Sa Ngiamsunthorn. Persistence of bounded solutions of parabolic equations under domain perturbation. J. Evol. Equ., 12(1):1-26, 2012.

[Sim78] B. Simon. A canonical decomposition for quadratic forms with applications to monotone convergence theorems. J. Funct. Anal., 28(3):377-385, 1978.

[Sta60] G. Stampacchia. Problemi al contorno ellitici, con dati discontinui, dotati di soluzionie hölderiane. Ann. Mat. Pura Appl. (4), 51:1-37, 1960.

[ZP05] V. Zhikov and S. Pastukhova. On operator estimates for some problems in homogenization theory. Russ. J. Math. Phys., 12(4):515-524, 2005. 